\title{
ON VITALI-HAHN-SAKS TYPE THEOREMS
}

\author{
BY BARBARA FAIRES ${ }^{1}$
}

Communicated by Robert Bartle, November 7, 1973

In recent years extensive work has been done on the Vitali-Hahn-Saks theorem and its relatives. Seever [13] considered the question of extending the Vitali-Hahn-Saks theorem to the case where the domain is a Boolean algebra which is not necessarily sigma complete. Brooks and Jewett [2] established results for a strongly bounded map defined on a Boolean sigma algebra of sets with values in a Banach space. Further generalizations to group-valued set functions have been studied by the Poznán school (see [5], [6], [7], [8], [9], [11], [12]). The work of all these authors is generalized herein to the case of strongly bounded maps defined on Boolean algebras with the Seever property and taking values in a Banach space. Some applications other than those considered herein and the final generalization to the group-valued case can be found in [10].

I wish to thank Professor J. Diestel for his advice and encouragement in the preparation of this paper. Also, I wish to express my gratitude to Professors R. E. Huff and J. J. Uhl, Jr. for many helpful discussions.

1. Notation and definitions. A Boolean algebra $\mathscr{B}$ has the property (I) if and only if for any sequences $\left\{x_{n}\right\}$ and $\left\{y_{n}\right\}$ in $\mathscr{B}$ satisfying $x_{n} \leqq y_{m}$ for all $n, m$, there exists $x \in \mathscr{B}$ such that $x_{n} \leqq x \leqq y_{n}$ for all $n$. This condition is equivalent to the condition: given any sequences $\left\{a_{n}\right\}$ and $\left\{b_{n}\right\}$ in $\mathscr{B}$ satisfying $a_{n} \wedge a_{m}=0, b_{n} \wedge b_{m}=0$ for $n \neq m$ and $a_{n} \wedge b_{m}=0$ for all $n, m$, there exists an element $a$ in $\mathscr{B}$ such that $a \geqq a_{n}$ and $b_{n} \wedge a=0$ for all $n$.

Unless signified otherwise, $\mathscr{B}$ will be used in this paper to denote a Boolean algebra with the property (I). The symbol $X$ denotes a Banach space and $X^{*}$ its Banach space dual.

A finitely additive $\mu: \mathscr{B} \rightarrow X$ is bounded whenever there exists $M>0$ such that $\|\mu(b)\| \leqq M$ for all $b \in \mathscr{B} ; \mu$ is said to be strongly bounded if $\left\|\mu\left(e_{n}\right)\right\| \rightarrow 0$ as $n \rightarrow \infty$ for each disjoint sequence $e_{1}, \cdots, e_{n}, \cdots$ of elements in $\mathscr{B}$. A sequence $\mu_{n}: \mathscr{B} \rightarrow X, n=1,2, \cdots$, is uniformly strongly bounded if for each disjoint sequence $\left\{e_{n}\right\} \subset \mathscr{B}, \lim _{n} \sup _{k}\left\|\mu_{k}\left(e_{n}\right)\right\|=0$. By grouping it is easy to see that if $\mu$ is strongly bounded and $\left\{e_{n}\right\} \subset \mathscr{B}$ is disjoint, then $\sum_{n=1}^{\infty} \mu\left(e_{n}\right)$ is an unconditionally convergent series in $X$. A map $\mu: \mathscr{B} \rightarrow X$ is

AMS (MOS) subject classifications (1970). Primary 46G99; Secondary 28A60.

${ }^{1}$ Supported in part by a Doctoral Fellowship granted by Kent State University. 
countably additive if for every disjoint sequence $\left\{e_{n}\right\} \subset \mathscr{B}$ with $\bigvee_{n} e_{n} \in \mathscr{B}$, the equality $\mu\left(\bigvee_{n} e_{n}\right)=\sum_{n} \mu\left(e_{n}\right)$ holds. The semivariation of $\mu$ on $b \in \mathscr{B}$, denoted by $\|\mu\|(b)$, is defined to be $\sup \{\|\mu(a)\|: a \in \mathscr{B}, a \leqq b\}$. It is easily shown that $\mu: \mathscr{B} \rightarrow X$ is strongly bounded if and only if $\|\mu\|: \mathscr{B} \rightarrow[0, \infty)$ is strongly bounded (though $\|\mu\|$ need not be additive).

\section{Main results.}

THEOREM 1. Let $\mu_{n}: \mathscr{B} \rightarrow X$ be finitely additive and strongly bounded for $n=1,2, \cdots$. If $\lim _{n} \mu_{n}(e)=0$ for each $e \in \mathscr{B}$, then $\left\{\mu_{n}: n \in N\right\}$ is uniformly strongly bounded.

Proof. Suppose not. Then there exists a sequence $\left\{e_{n}\right\}$ of disjoint elements of $\mathscr{B}$, a number $\varepsilon>0$, and a sequence $m_{1}<m_{2}<m_{3}<\cdots$ of positive integers (to simplify notation, assume $m_{n}=n$ ) such that for each $n \in N,\left\|\mu_{n}\left(e_{n}\right)\right\|>4 \varepsilon$.

Let $i_{1}=1$. Partition the set $N \backslash\{1\}$ into an infinite number of infinite disjoint sets $\pi_{n}^{1}, n=1,2,3, \cdots$. Utilizing property (I) we can choose a sequence $f_{n}^{1}, n=1,2, \cdots$, of disjoint elements in $\mathscr{B}$ such that:

(a $\left.\mathrm{a}_{1}\right) f_{n}^{1} \geqq e_{i}$ for all $i \in \pi_{n}^{1}, n=1,2, \cdots$;

(b) $f_{n}^{1} \wedge e_{i_{1}}=0, n=1,2, \cdots$;

(c) $f_{n}^{1} \wedge e_{j}=0$ for all $j \in(N \backslash\{1\}) \mid\left(\bigcup_{i=1}^{n} \pi_{i}^{1}\right)$.

As $\left\|\mu_{i_{1}}\right\|\left(f_{n}^{1}\right) \rightarrow 0(n \rightarrow \infty)$ there exists an $n_{1} \in N$ such that $\left\|\mu_{i_{1}}\right\|\left(f_{n_{1}}^{1}\right)<\varepsilon$. Choose $i_{2} \in \pi_{n_{1}}^{1}$ such that $i_{2}>i_{1}$ and $\left\|\mu_{i_{2}}\left(e_{i_{1}}\right)\right\|<\varepsilon / 4$. Partition the set $\pi_{n_{1}}^{1}\left\{\left\{i_{2}\right\}\right.$ into an infinite number of infinite disjoint sets $\pi_{n}^{2}, n=1,2, \cdots$. Again by property (I) there exists a sequence $f_{n}^{2}, n=1,2, \cdots$, of disjoint elements in $\mathscr{B}$ such that

(a $\left.\mathrm{a}_{2}\right) f_{n}^{2} \geqq e_{i}$ for all $i \in \pi_{n}^{2}, n=1,2, \cdots$;

(b) $f_{n}^{2} \wedge\left(e_{i_{1}} \vee e_{i_{2}}\right)=0, n=1,2, \cdots$;

(c) $f_{n}^{2} \wedge e_{j}=0$ for all $j \in\left(\pi_{n_{1}}^{1} \backslash\left\{i_{2}\right\}\right) \backslash\left(\bigcup_{i=1}^{n} \pi_{i}^{2}\right)$.

There exists an integer $n_{2} \in N$ such that $\left\|\mu_{i_{2}}\right\|\left(f_{n_{2}}^{2}\right)<\varepsilon$. Choose $i_{3} \in \pi_{n_{2}}^{2}$ such that $i_{3}>i_{2}$ and $\left\|\mu_{i_{3}}\left(e_{i_{1}}\right)\right\|,\left\|\mu_{i_{3}}\left(e_{i_{2}}\right)\right\|<\varepsilon / 8$. Proceed in this fashion to obtain a sequence $f_{n_{k}}^{k}=f_{k}, k=1,2, \cdots$, of elements of $\mathscr{B}$ and a sequence $i_{1}<i_{2}<\cdots$ of positive integers such that:

(1) $f_{n} \geqq e_{i_{k}}, k>n$;

(2) $f_{n} \wedge e_{i_{k}}=0,1 \leqq k \leqq n$;

(3) $\left\|\mu_{i_{n}}\right\|\left(f_{n}\right)<\varepsilon, n=1,2, \cdots$;

(4) $\left\|\mu_{i_{n}}\left(e_{i_{k}}\right)\right\|<\varepsilon / 2^{n}, 1 \leqq k<n$;

(5) $\left\|\mu_{i_{n}}\left(e_{i_{n}}\right)\right\|>4 \varepsilon, n=1,2, \cdots$.

Let $h_{n}=f_{n} \vee\left(\bigvee_{k=1}^{n} e_{i_{k}}\right)$. Then $h_{n} \geqq e_{i_{k}}$ for all $n, k$. Choose $c \in \mathscr{B}$ such that

$$
h_{n} \geqq c \geqq e_{i_{n}} \text { for all } n \text {. }
$$


Noticing that $\mu_{i_{n}}(c)=\mu_{i_{n}}\left(h_{n}-e_{i_{n}}\right)-\mu_{i_{n}}\left(h_{n}-c\right)+\mu_{i_{n}}\left(e_{i_{n}}\right)$, we have

$$
\begin{aligned}
\left\|\mu_{i_{n}}(c)\right\| \geqq & \left\|\mu_{i_{n}}\left(e_{i_{n}}\right)\right\|-\left\|\mu_{i_{n}}\left(h_{n}-e_{i_{n}}\right)\right\|-\left\|\mu_{i_{n}}\left(h_{n}-c\right)\right\| \\
= & \left\|\mu_{i_{n}}\left(e_{i_{n}}\right)\right\|-\left\|\mu_{i_{n}}\left[\left(f_{n} \vee\left(\bigvee_{k=1}^{n} e_{i_{k}}\right)\right) \wedge e_{i_{n}}^{\prime}\right]\right\| \\
& -\left\|\mu_{i_{n}}\left[\left(f_{n} \vee\left(\bigvee_{k=1}^{n} e_{i_{k}}\right)\right) \wedge c^{\prime}\right]\right\|
\end{aligned}
$$

which by (2) is

$$
\begin{aligned}
& \geqq\left\|\mu_{i_{n}}\left(e_{i_{n}}\right)\right\|-\left\|\mu_{i_{n}}\left(f_{n} \wedge e_{i_{n}}^{\prime}\right)\right\| \\
& \quad-\left\|\mu_{i_{n}}\left[\bigvee_{k=1}^{n}\left(e_{i_{k}} \wedge e_{i_{n}}^{\prime}\right)\right]\right\|-\left\|\mu_{i_{n}}\left(f_{n} \wedge c^{\prime}\right)\right\|-\left\|\mu_{i_{n}}\left[\bigvee_{k=1}^{n}\left(e_{i_{k}} \wedge c^{\prime}\right)\right]\right\| .
\end{aligned}
$$

Applying (2), (6) and the disjointness of the $e_{i_{k}}$ 's yields

$$
\begin{aligned}
\geqq & \left\|\mu_{i_{n}}\left(e_{i_{n}}\right)\right\|-\left\|\mu_{i_{n}}\left(f_{n}\right)\right\| \\
& \quad-\left\|\mu_{i_{n}}\left(e_{i_{n}}\right)\right\|-\cdots-\left\|\mu_{i_{n}}\left(e_{i_{n-1}}\right)\right\|-\left\|\mu_{i_{n}}\left(f_{n} \wedge c^{\prime}\right)\right\|,
\end{aligned}
$$

which by (5), (3) and (4) is $>4 \varepsilon-\varepsilon-(n-1) \varepsilon / 2^{n}-\varepsilon \geqq \varepsilon$. Since $\left\|\mu_{i_{n}}(c)\right\|>\varepsilon$ holds for infinitely many $n, \lim _{n} \mu_{n}(c) \rightarrow 0$, a contradiction.

The proofs of some of the corollaries yielded by Theorem 1 are, for the most part, minor alterations to proofs presented elsewhere; in these cases the appropriate references are given.

COROllary 1 [2, COROLlaRy 1.2]. Let $\mu_{n}: \mathscr{B} \rightarrow X$ be finitely additive and strongly bounded for $n=1,2, \cdots$. If $\lim _{n} \mu_{n}(e)=\mu(e)$ exists for each $e \in \mathscr{B}$, then $\mu$ is strongly bounded and the $\mu_{n}, n=1,2, \cdots$, are uniformly strongly bounded.

CoROllaRY 2. Let $\mu_{n}: \mathscr{B} \rightarrow X$ be countably additive for $n=1,2, \cdots$. If $\lim _{n} \mu_{n}(e)=\mu(e)$ exists for each $e \in \mathscr{B}$, then $\mu$ is countably additive and the $\mu_{n}, n=1,2, \cdots$, are uniformly countably additive.

Corollary 3 [3, TheOREM 1.6]. Let $X$ be any separable Banach space and let $\mu: \mathscr{B} \rightarrow X$ be bounded and finitely additive. Then $\mu$ is strongly bounded.

Another corollary is the following result proved differently by $\mathbf{N}$. J. Kalton in an unpublished manuscript.

COROLlaRY 4. Let $X$ be a weakly compactly generated Banach space and let $\mu: \mathscr{B} \rightarrow X$ be bounded and finitely additive. Then $\mu$ is strongly bounded. 
Proof. Let $\left\{e_{n}\right\}$ be a disjoint sequence in $\mathscr{B}$ and let $\left[\mu\left(e_{n}\right)\right]=X_{0}$ denote the closed linear span of $\left\{\mu\left(e_{n}\right): n \in N\right\}$. Then $X_{0}$ is a separable subspace of the weakly compactly generated space $X$; hence by a result of Amir and Lindenstrauss [1, Lemma 4], there is a separable subspace $Y$ of $X$ such that $X_{0} \subset Y$ and $Y$ is complemented in $X$. Suppose $P: X \rightarrow Y$ is the projection. Then Corollary 1.2 yields $P \circ \mu\left(e_{n}\right) \rightarrow 0, n \rightarrow \infty$. But $P \circ \mu\left(e_{n}\right)=\mu\left(e_{n}\right)$ for each $n$. Therefore, $\mu$ is strongly bounded.

Corollary 5 [4, Corollary 5]. Let $\mu_{n}: \mathscr{B} \rightarrow X$ be strongly bounded for $n=1,2, \cdots$. Suppose $\mu(e)=$ weak-limit $_{n} \mu_{n}(e)$ exists for each $e \in \mathscr{B}$. Then $\mu$ is strongly bounded.

Proof. The boundedness of $\mu$ follows from the Banach-Steinhaus theorem and Corollary 1.1 applied to the functions $f \mu_{n}, f \mu$ where $f \in X^{*}$. For each $n$ let $B_{n}=\mu_{n}(\mathscr{B})$ and let $Y$ be the closed linear span of $\bigcup_{n} B_{n}$. By the definition of $\mu$ and Mazur's theorem we have $\mu(\mathscr{B}) \subset Y$. We claim that $Y$ is weakly compactly generated.

For each $n$, let $M_{n}=\sup \left\{\left\|\mu_{n}(b)\right\|: b \in \mathscr{B}\right\}$. Let $B=\bigcup_{n} B_{n} /\left(n \cdot M_{n}\right)$. The closed linear span of $B$ is $Y$ and $B$ is relatively weakly compact. To see the last assertion, let $\left\{y_{n}\right\}$ be a sequence in $B$. Since each $\mu_{n}$ is strongly bounded, $B_{n}$, and hence $B_{n} /\left(n \cdot M_{n}\right)$, is relatively weakly compact [14]. So if $\left\{y_{n}\right\}$ returns infinitely often to one of the $B_{n} /\left(n \cdot M_{n}\right)$ 's, we can extract a weakly convergent subsequence. If $\left\{y_{n}\right\}$ does not return infinitely often to any $B_{n} /\left(n \cdot M_{n}\right)$ then there exist strictly increasing sequences $\left(m_{k}\right)$ and $\left(n_{k}\right)$ of positive integers such that $y_{m_{k}} \in B_{n_{k}} /\left(n_{k} \cdot M_{n_{k}}\right)$ for each $k$. It follows that $\left\|y_{m_{k}}\right\| \leqq 1 / n_{k} \rightarrow 0$ as $k \rightarrow \infty$. Thus $\left\{y_{n}\right\}$ has a norm convergent subsequence.

With the proof proceeding as in [2, Theorem 3] we have the following Vitali-Hahn-Saks theorem.

THEOREM 2. Let $\mu_{n}: \mathscr{B} \rightarrow X$ be finitely additive and strongly bounded, for $n=1,2, \cdots$. Suppose $v$ is a nonnegative monotone set function defined on $\mathscr{B}$ and each $\mu_{n} \ll \nu$. Assume that $\lim _{n} \mu_{n}(e)$ exists for each $e \in \mathscr{B}$. Then $\lim _{v(e) \rightarrow 0}\left\|\mu_{n}(e)\right\|=0$ uniformly in $n$.

\section{REFERENCES}

1. D. Amir and J. Lindenstrauss, The structure of weakly compact sets in Banach spaces, Ann. of Math. (2) 88 (1968), 35-46. MR 37 \#4562.

2. J. K. Brooks and R. S. Jewett, On finitely additive vector measures, Proc. Nat. Acad. Sci. U.S.A. 67 (1970), 1294-1298. MR 42 \#4697.

3. J. Diestel, Applications of weak compactness and bases to vector measures and vectorial integration, Rev. Roumaine Math. 18 (1972), 211-224.

4. - Grothendieck spaces and vector measures, Vector and Operator Valued Measures and Applications, Academic Press, New York, 1973. 
5. L. Drewnowski, Control submeasures and control measures, Studia Math. (to appear).

6. —- Decompositions of set functions, Studia Math. 48 (1973), 23-48.

7. — Topological rings of sets, continuous set functions, integration. I, Bull. Acad. Polon. Sci. Sér. Sci. Math. Astronom. Phys. 20 (1972), 269-276. (Russian) MR 46 \#5558.

8. - Topological rings of sets, continuous set functions, integration. II, Bull. Acad. Polon. Sci. Sér. Sci. Math. Astronom. Phys. 20 (1972), 277-286. (Russian) MR 46 \#5558.

9. - Topological rings of sets, continuous set functions, integration. III, Bull. Acad. Polon. Sci. Sér. Sci. Math. Astronom. Phys. 20 (1972), 439-445.

10. Barbara Faires, Grothendieck spaces and vector measures, Ph.D. Dissertation, Kent State University, Kent, Ohio, August, 1974.

11. I. Labuda, On some generalizations of the theorems of Nikodym and of Vitali-HahnSaks, Bull. Acad. Polon. Sci. Sér. Sci. Math. Astronom. Phys. 20 (1972), 447-456.

12. ——, Sur le Théorème de Bartle-Dunford-Schwartz, Bull. Acad. Polon. Sci. Sér. Sci. Math. Astronom. Phys. 20 (1972), 549-553.

13. G. L. Seever, Measures on F-spaces, Trans. Amer. Math. Soc. 133 (1968), 267280. MR 37 \#1976.

14. J. J. Uhl, Jr., Extensions and decompositions of vector measures, J. London Math. Soc. (2) 3 (1971), 672-676. MR 44 \#4181.

Department of Mathematics, Kent State University, Kent, Ohio 44240 